1. Reginaldo, R. \& Sitko, M. L. IAU Circ. No. 3276 (1978).

2. Baldinelli, L. et al. IAU Circ. No. 3278 (1978).

3. Baroni, S., Cavagna, M., Margrave, T. E. \& Morgan, J. IAU Circ. No. 3281 (1978)

4. Vogt, S., Tull, R. G. \& Kelton, P. Appl. Opt. 17, 574 (1978).

5. Hobbs, L. Astrophys. J. 191, 381 (1974).

6. Ake, T. B., Duerbeck, H. W., Lanning, H. \& Machnaki, S. W. IAU Circ. No. 3272 (1978).

7. Deutschman, W. A., Davis, R. J. \& Schild, R. E. Astrophys. J. Suppl. 30, 97 (1976).

8. McLaughlin, D. B Publ astr. Soc. Pac 57, 69 (1945).

9. Andrews, P. J. et al. IAU Circ. No. 3268 (1978).

\section{Is Crab nebula a disrupted helium star?}

WE propose here that the Crab nebula was produced by the explosion of a helium star in a close binary already containing a neutron star. This explosion would be caused by a complete spiralling of the neutron star into the helium star, which was totally disrupted ${ }^{1}$. The neutron star would be spun up in the process and be identified with PSR0532.

Arnett has pointed out that the overabundance of helium with respect to hydrogen in the Crab filaments ${ }^{2}$ is compatible with a presupernova helium core of mass $4 M_{\odot}$ (ref. 3) which corresponds to a main sequence mass of $12 M_{\odot}$ (ref. 4). Chevalier ${ }^{5}$, on examining old Chinese records, present day nebula and the embedded pulsar, finds that all available information is consistent with the Crab having a massive progenitor. The fact that the motion of the Crab pulsar is directed away from the I Gem association has led to the suggestion ${ }^{6}$ that the progenitor of the Crab was a runaway star from I Gem. According to this model, the first supernova explosion in a close binary in I Gem disrupted the binary releasing a neutron star (identified with PSR0527) and a normal star of mass $12 M_{\odot}$ which later exploded to give the Crab nebula and the pulsar.

The model of a massive progenitor for the Crab as discussed above presents some problems. First, detailed work on the evolution of close binaries in the context of compact X-ray sources has shown (see ref. 1) that the first supernova explosion in a mass transfer binary is unlikely to disrupt the system. (If the binary is sufficiently wide so that mass transfer does not take place, the velocity of the normal star at the time of the disruption of the binary-equal to its orbital velocity-would be very small). Thus we are faced with the problem of placing a massive progenitor for Crab at $|z|=200 \mathrm{pc}$. Second, to avoid an observable overabundance of heavy elements in the Crab filaments, it must be assumed ${ }^{3}$ that the explosion of the helium star leaves behind a $1.45 M_{\odot}$ neutron star core. Now this value is substantially larger than $0.5 M_{\odot}$ estimated for the Crab pulsar on the basis of detailed models of neutron stars and data on micro- and macroglitches ${ }^{7}$. Third, there is the crucial problem of the mass. For the nebula a total mass of $\sim 1 M_{\odot}$ has been estimated from the absolute intensities of emission lines ${ }^{8}$. On the other hand, Davidson et $a l^{9}$ conclude that a nebular mass in the range 0.3-10 $M_{\odot}$ would not be inconsistent with observations, most of the mass being in dense neutral cores of filaments. However, the fact that bright and faint filaments have been accelerated equally, argues against the presence of too much neutral matter ${ }^{10}$. The mass of the nebula is therefore unlikely to be very much greater than $1 M_{\odot}$. Thus the problem is that if the Crab is a result of the supernova explosion of a massive star, where is the remaining mass?

These problems are solved if we assume that the progenitor of the Crab was a mass transfer binary. It is then obvious that the event of 1054 could not have been the first supernova explosion, because such an explosion would leave the binary intact. In other words the progenitor binary of the Crab must have passed through a Cen X3 type X-ray stage. The evolution of a normal massive star in a close binary containing a neutron star is characterised by a tremendous loss of mass and angular momentum ${ }^{1-13}$. The normal star expands and engulfs the neutron star, which leads to a rapid shrinkage of the orbit ${ }^{14}$. The spiralling in of the neutron star terminates when only the helium core of the companion is left, the hydrogen rich envelope having been blown off ${ }^{13}$. For example ${ }^{15}$, starting with an X-ray binary consisting of a $1 M_{\odot}$ neutron star and a $15 M_{\odot}$ normal star, we may arrive at a system consisting of a $1 M_{\odot}$ neutron star and a $3 M_{\odot}$ helium star and having a period of $\sim 1.5 \mathrm{~h}$ corresponding to a separation of about $1 R_{\odot}$.

It has implicitly been assumed ${ }^{16,17}$ that the helium star now evolves as a single star, so that its explosion in most cases distrupts the binary releasing two neutron stars, the old spun up neutron star manifesting itself as a type $\mathrm{L}$ pulsar and the newborn as a type D pulsar. However, conditions may be such that the helium star does not evolve as an isolated star. A $3 M_{\odot}$ helium star has a radius of $1.4 R_{\odot}$ at the onset of carbon ignition and of $4.5 R_{\odot}$ at the onset of neon burning ${ }^{18}$. Thus if the separation between the two stars is sufficiently small, at some point in the evolution of the helium star the neutron star would be engulfed, triggering off a new spiralling in, which may totally disrupt the helium star and spin up the neutron star to very short periods ${ }^{1}$.

According to our hypothesis, the Crab nebula is such a disrupted helium star and the Crab pulsar, a spun up old neutron star (a type L pulsar). This model has many attractive features. It provides a helium star for explosion without an embarassingly large mass for the nebula. It explains the observed overabundance of helium without postulating an excessively heavy neutron star or an unwanted excess of heavy elements. In this context it is relevant that Arnett $^{3}$ finds a better agreement between estimated ${ }^{3}$ and inferred ${ }^{2}$ abundances if interstellar matter is swept up rather than an old envelope (as would be the case if the exploding star were single).

The spun up neutron star in our model would move with a velocity equal to the centre of mass velocity the progenitor binary had acquired at the time of the formation of the neutron $\operatorname{star}^{1}$. (In refs 16,17 it was assumed that a type $L$ pulsar moves in the orbital plane of the progenitor binary). In the light of the above discussion, the fact that the motion of the Crab pulsar is directed away from the I Gem association permits a more plausible explanation (see ref. 6). The progenitor of the Crab was a massive close binary in I Gem. The first supernova explosion did not disrupt the binary, rather imparted the centre of mass a velocity of about $125 \mathrm{~km} \mathrm{~s}^{-1}$. In about the $3 \mathrm{Myr}$ between the first and the second explosion, the binary covered a distance of $\sim 380 \mathrm{pc}-$ crossing the galactic plane-and appearing at the site of the explosion of 1054. (Note that in the model of Gott et al. ${ }^{6}$, the Crab pulsar did not acquire any velocity as a result of the supernova explosion).

The earlier suggestion ${ }^{17}$ that PSR0532 is a type D pulsar and the associated type L pulsar should still be embedded in Crab nebula appears to be less attractive because of the problem of the abundance of heavy elements discussed above.

\section{Universitäts-Sternwarte, Geismarlandstraße 11, 34 Göttingen, FRG}

Received 6 October; accepted 22 November 1978.

Permanent address: Indian Institute of Astrophysics, Bangalore 34, India.

1. Heuvel, E. P. J. van den. A. N.Y. Acad. Sci. 302, 14-35 (1977)

2. Davidson, K. Astrophys. J. 186, 223-231 (1973).

3. Arnett, W. D. Astrophys. J. 195, 727-733 (1975).

4. Arnett, W. D. Astrophys. J. 219, 1008-1016 (1978)

5. Chevalier, R. A. in Supernovae (ed. Schramm, D. N.) (Dordrecht, Reidel, 1977).

6. Gott, J. R. III, Gunn, J. E. \& Ostriker, J. P. Astrophys. J. Lett. 160, L91-L95 (1970).

7. Shahan, J., Pines, D. \& Ruderman, M. A. A. N.Y. Acad. Sci. 224, 190-205 (1973).

8. Woltjer, L. A. Rev. Astr. Astrophys. 10, 129-158 (1972)

9. Davidson, K., Crane, P. \& Chincarini, G. Astr. J. 79, 791-796 (1974).

0. Trimble, V. \& Woltjer, L. Astrophys. J. Lett. 163, L97-L98 (1971).

11. Paczynski, B. Proc. IAU Symp. 73, 75-80 (1976).

2. De Greve, J.P., De Loore, C. \& Sutantyo, W. Astrophys. Space Sci. 38, 301-312 (1975)

13. Taam, R. E. Bodenheimer, P. \& Ostriker, J. P. Astrophys. J. 222, 269-280 (1978).

14. Sparks, W. M. \& Stecher, T. P. Astrophys. J. 188, 149-153 (1974).

15. Heuvel, E. P. J. van den \& De Loore, C. Astr. Astrophys. 25, 387-395 (1973)

16. Kochhar, R. K. Nature 267, 231 (1977); 270, 38-39 (1977).

17. Kochhar, R. K. Mem. Soc. Astr. Isal. (in the press).

18. Arnett, W. D. in Enrico Fermi School on the Physics of Compact Objects, Varenna (North Holland, Amsterdam, 1975) 